\title{
ISOMORPHISM OF WEIGHTED TREES AND STANLEY'S ISOMORPHISM CONJECTURE FOR CATERPILLARS
}

\author{
MARTIN LOEBL AND JEAN-SÉBASTIEN SERENI
}

\begin{abstract}
This paper contributes to a programme initiated by the first author: 'How much information about a graph is revealed in its Potts partition function?'. We show that the $W$-polynomial distinguishes non-isomorphic weighted trees of a good family. The framework developed to do so also allows us to show that the $W$-polynomial distinguishes non-isomorphic caterpillars. This establishes Stanley's isomorphism conjecture for caterpillars, an extensively studied problem.
\end{abstract}

\section{INTRODUCTION}

Consider the following data set $D(T)$ associated with a tree $T$ : for every integer $n$ and every partition $P$ of $n$, we are given the number of subsets $X$ of edges of $T$ such that $P$ is equal to the multiset formed by the orders of the components of $T-X$. Note that this number is 0 if $n$ is not the number of vertices of $T$. Note also that if $P$ is composed of $t$ integers, the corresponding subsets $X$, if any, all have cardinality $t-1$. For instance, one can determine the number of vertices of $T$ by checking, for each positive integer $n$, whether the trivial partition $\{n\}$ returns a non-zero value (which, necessarily, will be 1 ). Once the number $n$ of vertices of $T$ is known, the number of leaves of $T$ is precisely the number returned by the partition $\{n-1,1\}$, which corresponds to the number of edges $e$ such that $T-e$ has one component of order 1 . The problem is to know whether this information distinguishes non-isomorphic trees. In other words, if $T$ and $T^{\prime}$ are two trees such that $D(T)=D\left(T^{\prime}\right)$, is it true that necessarily $T$ and $T^{\prime}$ are isomorphic? That such a reconstruction is always possible was suggested by different authors. We note that there could be non-constructive proofs of the statement. Thus it is a different (harder) problem to be able to effectively recover the tree $T$ from the knowledge of $D(T)$. We explain in subsections 2.1 2.2 and 2.3 why studying the strength of the information contained in $D(T)$ for an arbitrary tree $T$ helps to understand the strength of the partition function of the Potts model in a magnetic field, for general graphs.

1.1. State of the Art. Extensive efforts were dedicated (personal communication with Noble) to proving that $D(T)$ distinguishes non-isomorphic caterpillars - a caterpillar is a tree where all edges not incident with a leaf form a path, and a leaf is a vertex of degree one. Part of the Ph.D. thesis of Zamora [18] (under the supervision of M. L.) is dedicated to this problem. In addition, Aliste-Prieto and Zamora 1, established the statement restricted to the class of proper caterpillars: a caterpillar is proper if every vertex is a leaf or adjacent to a leaf. Prior to that, partial results had been obtained by Martin, Morin and Wagner 9] who had established the

Date: June 19, 2018.

2010 Mathematics Subject Classification. 05C31, 05C60.

Key words and phrases. $W$-polynomial, tree, graph reconstruction, graph isomorphism, $U$-polynomial, Stanley's isomorphism conjecture, Potts partition function.

This work was done within the scope of the International Associated Laboratory STRUCO.

The authors were partially supported by the Czech Science Foundation under the contract number P202-1321988S (M. L.) and by P.H.C. Barrande 31231PF of the French M.A.E. (J.-S. S.). 
statement for a subclass of proper caterpillars (where no two non-leaf vertices are adjacent to the same number of leaves) and also to the class of spiders, which is composed of all trees with a unique vertex of degree greater than two. Other related results were obtained by Orellana and Scott [12, Smith, Smith and Tian [15] or can be found in the undergraduate thesis by Fougere [5] and the MSc thesis by Morin [10]. Finally, Hell and Ji [6] have verified by computer that Stanley's isomorphism conjecture [16], which we present in Subsection 2.2 is true for trees with at most 29 vertices. Previously, Russel has verified by computer that Stanley's isomorphism conjecture is true for trees with at most 25 vertices (the code is available at https://github.com/keeler/csf) and it was reported (see [9, p. 238]) that Tan verified it for trees with at most 23 vertices.

1.2. Main Contribution. We solve affirmatively Stanley's isomorphism conjecture restricted to the class of caterpillars. We also investigate a weighted version of the problem, bearing in mind its connections with graph polynomials, graph colouring and the Potts model. First we summarise the background and motivations.

\section{Motivation}

In this section we summarise the background (the Noble and Welsh conjecture and the Stanley conjecture) and describe our motivation.

2.1. The Noble and Welsh Conjecture. Motivated by the combinatorial aspects of the relationship between chord diagrams and Vassiliev invariants of knots, Noble and Welsh [11] introduced a polynomial of weighted graphs, the $W$-polynomial, which includes several specialisations in combinatorics, such as the Tutte polynomial, the matching polynomial (of ordinary graphs) and the polymatroid polynomial of Oxley and Whittle [13. We need to introduce some terminology to define $W$.

A weighted graph is a graph $G=(V, E)$ together with a function $w: V \rightarrow \mathbf{Z}^{+}$. The weight of a subset $V^{\prime}$ of vertices is $w\left(V^{\prime}\right):=\sum_{v \in V^{\prime}} w(v)$. If $A \subseteq E$, we let $c_{V}(A)$ be the number of components of the graph $(V, A)$, where we may omit the subscript when there is no risk of confusion. Further, let $n_{1}, \ldots, n_{c(A)}$ be the weights of the vertex sets of these components, listed in decreasing order: $n_{1} \geqslant \cdots \geqslant n_{c(A)}$. We write $x(A)$ to mean $\prod_{i=1}^{c(A)} x_{n_{i}}$. Let

$$
W_{G}\left(z, x_{1}, x_{2}, \ldots\right):=\sum_{A \subseteq E} x(A)(z-1)^{|A|-|V|+c(A)} .
$$

In particular, $W_{G}$ depends on $z$ if and only if $G$ contains a cycle [11, Proposition 5.1]. Unlike the Tutte polynomial, the $W$-polynomial is \#P-hard to compute even for trees [11, Theorems 7.3 and 7.12] and for complete graphs [11, Theorems 7.11 and 7.14].

In the case of unweighted graphs, which corresponds here to the weight function $w$ being identically 1 , Noble and Welsh refer to the $W$-polynomial as the $U$-polynomial. While computing $W$ is hard for complete graphs, Annan [2] proved that $U_{K_{n}}\left(z, x_{1}, x_{2}, \ldots\right)$ can be computed in polynomial time, which is also the case for the Tutte polynomial. However, $U$ also exhibits differences with the Tutte polynomial: while finding two non-isomorphic graphs with the same Tutte polynomial is easy, the same problem is harder for $U$. Brylawski [4] found two nonisomorphic graphs with the same polychromate, and Sarmiento [14 proved that the $U$-polynomial is equivalent to Brylawski's polychromate. But the question remains open for trees: does the $U$-polynomial distinguishes non-isomorphic trees? That this is the case became known as the Noble and Welsh conjecture. This is clearly equivalent to our initial question: 'Does $D(T)$ distinguish non-isomorphic trees?'

Noble and Welsh demonstrated the $U$-polynomial to be equivalent to the symmetric function generalisation of the chromatic polynomial, a function introduced by Stanley [16]. 
2.2. Stanley's isomorphism Conjecture. To introduce Stanley's isomorphism conjecture let us first define graph colouring. A colouring of a graph $G=(V, E)$ is a mapping $s: V \rightarrow \mathbf{N}^{+}$. We define $b(s)$ to be the number of monochromatic edges in $s$, that is, the number of edges $u v$ such that $s(u)=s(v)$. The mapping $s$ is a $k$-colouring if $s(V) \subseteq\{1, \ldots, k\}$ and $s$ is proper if $b(s)=0$, that is, $s(u) \neq s(v)$ whenever $u$ and $v$ are two adjacent vertices of $G$. We let $\operatorname{Col}(G ; k)$ be the set of proper $k$-colourings of $G$ and $\operatorname{Col}(G)$ be the set of all proper colourings of $G$.

In the mid 1990s, Stanley [16] introduced the symmetric function generalization of the chromatic polynomial, defined to be

$$
X_{G}\left(x_{1}, x_{2}, \ldots\right):=\sum_{s \in \operatorname{Col}(G)} \prod_{v \in V} x_{s(v)}
$$

This is a homogeneous symmetric function in $\left(x_{1}, x_{2}, \ldots\right)$ of degree $|V|$. As one might expect, $X_{G}$ does not distinguish non-isomorphic graphs: there exist two non-isomorphic graphs on 5 vertices with the same function $X$. However, Stanley [16] asked whether the polynomial $X_{G}$ distinguishes non-isomorphic trees. The assertion that it does became known as Stanley's isomorphism conjecture.

Further, Stanley [17] later initiated the study of a common generalisation of $X$ and the Tutte polynomial, namely the symmetric function generalisation of the bad colouring polynomial, defined for every graph $G=(V, E)$ by

$$
X_{G}\left(t, x_{1}, x_{2}, \ldots\right):=\sum_{s: V \rightarrow \mathbf{N}^{+}}(1+t)^{b(s)} \prod_{v \in V} x_{s(v)} .
$$

Note that the sum runs over all colourings of $G$, not only the proper ones. Noble and Welsh 11, Theorem 6.2] proved $X_{G}\left(t, x_{1}, x_{2}, \ldots\right)$ to be equivalent to the $U$-polynomial of $G$.

2.3. Loebl's Conjectures. Loebl 8 introduced the $q$-chromatic functions. Let $k \in \mathbf{N}$. The q-chromatic function of a graph $G=(V, E)$ is

$$
M_{G}(k, q):=\sum_{s \in \operatorname{Col}(G ; k)} q^{\sum_{v \in V} s(v)} .
$$

It is known 8 , that

$$
M_{G}(k, q)=\sum_{A \subset E}(-1)^{|A|} \prod_{C \in \mathscr{C}(A)}(k)_{q^{|C|}},
$$

where the quantum integer $(k)_{r}$ is $r^{k-1}+\cdots+r+1$ and $\mathscr{C}(A)$ is the set of components of the spanning subgraph $(V, A)$ while $|C|$ is the number of vertices in the component $C$. Moreover Loebl also introduced the $q$-dichromate, defined as

$$
B_{G}(x, y, q):=\sum_{A \subset E} x^{|A|} \prod_{C \in \mathscr{C}(A)}(y)_{q^{|C|}} .
$$

Loebl [8] conjectured the following.

- The $q$-dichromate is equivalent to the $U$-polynomial.

- The $U$-polynomial distinguishes non-isomorphic chordal graphs.

There could be a close link between the latter conjecture and that of Stanley: chordal graphs have a very distinguished tree structure. Indeed, a folklore theorem [3] states that the class of chordal graphs is precisely the class of intersection graphs of subtrees of a tree, that is, for each chordal graph $G$, there exists a tree $T$ and a mapping $f$ that assigns to each vertex of $G$ a subtree $T$ such that: two vertices $u$ and $v$ of $G$ are adjacent if and only if $f(u) \cap f(v) \neq \varnothing$.

The motivation for Loebl's conjectures is formula 2.2$)$ below, which connects the $k$-state Potts model partition function and the $q$-dichromate. 
The Potts model. We consider a standard model where magnetic materials are represented as lattices: vertices are atoms and weighted edges are nearest-neighbourhood interactions. We assume that each atom has one out of $k$ possible magnetic moments, for a fixed positive integer $k$. Thus we set $S:=\{0, \ldots, k-1\}$. Every element of $S$ is called a spin. A state of a graph $G=(V, E)$ is then an assignment of a single spin to each vertex of $G$, that is, a function $s: V \rightarrow S$. We assume that all the coupling constants (nearest-neighbourhood interactions) are equal to a constant $J$. For each state $s$, the Potts model energy of the state $s$ is defined to be $E\left(P^{k}\right)(s):=\sum_{u v \in E} J \delta(s(u), s(v))$ where, as is customary, $\delta$ is the Kronecker delta function defined by $\delta(a, b):=1$ if $a=b$ and $\delta(a, b):=0$ otherwise. The $k$-state Potts model partition function is

$$
\sum_{s: V \rightarrow S} M(s, J) e^{E\left(P^{k}\right)(s)}
$$

where $M(s, J)$ is a function describing the magnetic field contribution.

Loebl proved that for each real $J$,

$$
B_{G}\left(e^{J}-1, k, q\right)=\sum_{s: V \rightarrow S} q^{\sum_{v \in V} s(v)} e^{E\left(P^{k}\right)(s)} .
$$

This means that the $q$-dichromate specializes to the $k$-state Potts model partition function with a certain magnetic field contribution.

Recently a variant of the $q$-dichromate, $B_{r, G}(x, k, q)$, was proposed by Klazar, Loebl and Moffatt [7]:

$$
B_{r, G}(x, k, q):=\sum_{A \subseteq E} x^{|A|} \prod_{C \in \mathscr{C}(A)} \sum_{i=0}^{k-1} r^{|C| q^{i}} .
$$

They established that if $(k, r) \in \mathbf{N}^{2}$ with $r>1$ and $x:=\mathrm{e}^{\beta J}-1$, then

$$
B_{r, G}(x, k, q)=\sum_{\sigma: V \rightarrow S} \mathrm{e}^{\beta \sum_{u v \in E(G)} J \delta(\sigma(u), \sigma(v))} r^{\sum_{v \in V} q^{\sigma(v)}} .
$$

Hence $B_{r, G}(x, k, q)$ is the $k$-state Potts model partition function with magnetic field contribution $r^{\sum_{v \in V} q^{\sigma(v)}}$. They also proved $B_{r, G}$ to be equivalent to $U_{G}$, which can be seen as a first step towards Loebl's programme:

The polynomial $U_{G}$ is equivalent to the Potts partition function of $G$ with a magnetic field contribution.

A well-known fact is that the isomorphism problem for general graphs is equivalent to the isomorphism problem restricted to chordal graphs: given a graph $G=(V, E)$, consider the chordal graph $G^{\prime}=\left(V^{\prime}, E^{\prime}\right)$ so that $V^{\prime}:=V \cup E$ and $E^{\prime}=\left(\begin{array}{c}V \\ 2\end{array}\right) \cup\{\{u, e\},\{v, e\}:\{u, v\}=e \in E\}$. It clearly holds that $G$ and $H$ are isomorphic if and only if $G^{\prime}$ and $H^{\prime}$ are isomorphic. It thus seems particularly interesting to determine whether the $U$-polynomial does distinguish non-isomorphic chordal graphs, as conjectured by Loebl. If true, we would obtain a surprising conclusion:

The Potts partition function with a magnetic field contribution contains essentially (modulo a simple preprocessing) all the information about the underlying graph.

In that respect, it seems natural to study weighted trees. The tree mentioned in the characterisation of the class of chordal graphs can be chosen to be a clique-tree, where the vertices of the tree are the maximal cliques of the graph. Now, if $v$ is a vertex of a weighted tree with weight $w(v)$, one can think of $v$ as a clique of order $w(v)$, thus obtaining an unweighted chordal graph. This is what motivates working in the (seemingly harder) setting of weighted trees. 
2.4. Main Results. Two weighted graphs are isomorphic if there is an isomorphism of the graphs that preserves the vertex weights. We also consider weighted trees rooted at a vertex: an isomorphism between rooted weighted trees preserves the weights by definition, but may not preserve the roots. If it does preserve the roots, then it is an r-isomorphism. (In particular, two rooted weighted trees that are r-ismomorphic are isomorphic but the converse is not necessarily true.)

The first purpose of this work is to prove that the $W$-polynomial distinguishes non-isomorphic weighted trees when restricting to collections of weighted trees satisfying some properties made precise later. We call any such collection a good family. We consider this result as a first observation towards understanding Stanley's isomorphism conjecture for the class of chordal graphs; even though we do not know natural examples of good families of weighted trees which were studied before. We remark that the $W$-polynomial does not distinguish general weighted trees; a simple example consists of two paths with weight sequences $1,2,1,3,2$ and 1,3,2,1,2.

Let $(T, w)$ be a weighted tree. We write $V(T)$ and $E(T)$ for the vertex set and the edge set of $T$, respectively. We define $\operatorname{Ex}(T)$ to be the multi-set composed of all the vertex weights (with multiplicities) of $T$. If $e \in E(T)$, then $T-e$ is the disjoint union of two trees, which we consider to be weighted and rooted at the endvertex of $e$ that they contain. A rooted weighted tree $\left(S, w_{S}\right)$ is a shape of $(T, w)$ if $2 \leqslant|V(S)| \leqslant|V(T)|-2$ and there exists an edge $e \in E(T)$ such that $S$ is one of the two components of $T-e$; moreover $w_{S}$ is the restriction of $w$ to the vertex set of $S$. We consider $S$ to be rooted at the end-vertex of $e$. We usually shorten the notation and write $S$ for the shape $\left(S, w_{S}\right)$. In a tree, a vertex of degree one is called a leaf.

Definition 2.1. A set $\mathcal{T}$ of weighted trees $(T, w)$ is good if it satisfies the following properties.

(1) If a vertex of $T$ is adjacent to a leaf, then all its neighbours but possibly one are leaves.

(2) If $v$ is a leaf or has a neighbour that is a leaf, then $w(v)=1$.

(3) Let $(T, w),\left(T^{\prime}, w^{\prime}\right) \in \mathcal{T}$ and let $S$ be a shape of $T$ and such that $w(S) \leqslant w(T) / 2$. Let $S^{\prime}$ be a shape of $T^{\prime}$ such that $\operatorname{Ex}\left(S^{\prime}\right)=\operatorname{Ex}(S)$. Then $S^{\prime}$ and $S$ are r-isomorphic.

Theorem 1. The $W$-polynomial distinguishes non-isomorphic weighted trees in any good set.

Our proof of Theorem 1 is not constructive in the sense that we are not able to reconstruct the weighted tree $(T, w)$ from $W_{(T, w)}$. The difficulty in proving the theorem is that while the main defining property of a good family is about shapes, the $W$-polynomial does not "see" shapes.

However, shapes turn out to be a useful and rather powerful notion: it allowed us to unlock the case of general caterpillars, thereby confirming Stanley's isomorphism conjecture for the class of (general) caterpillars.

Theorem 2. Each caterpillar can be reconstructed from its $U$-polynomial.

Note that Theorem 2, contrary to Theorem 1, allows for a full reconstruction of the tree.

\section{The Structure of the Proofs}

We write down a procedure and with its help prove both theorems. The rest of the paper then describes our realisation of the procedure. We fix a good set of weighted trees and, from now on, we say that a weighted tree is good if it belongs to this set.

A $j$-form is an r-isomorphism class of rooted weighted trees with total weight $j$. Thus a $j$-form $F$ is a collection of r-isomorphic rooted weighted trees and, viewing a shape of a tree $T$ as a rooted weighted tree, a shape can belong to a $j$-form. Note in particular that two shapes $S$ and $S^{\prime}$ of a weighted tree belong to the same $j$-form for some $j$ if and only if $S$ and $S^{\prime}$ are r-isomorphic. We start with two observations. 
Observation 3.1. Let $T_{1}$ and $T_{2}$ be two shapes of a tree $T$ such that $w\left(T_{1}\right)+w\left(T_{2}\right) \leqslant w(T)$. Then either $T_{1} \subseteq T_{2}$ or $T_{2} \subseteq T_{1}$ or $T_{1} \cap T_{2}=\varnothing$.

Proof. For $k \in\{1,2\}$ let $e_{k}$ be the edge of $T$ associated to $T_{k}$, that is, $T_{k}$ is a component of $T-e_{k}$. If $e_{1}=e_{2}$, then either $T_{1}=T_{2}$ or $T_{1} \cap T_{2}=\varnothing$. Assume that $e_{1} \neq e_{2}$. Then either $e_{2} \in E\left(T_{1}\right)$ or $e_{2} \in E\left(T-T_{1}\right)$. If $e_{2} \in E\left(T-T_{1}\right)$, then either $T_{1} \subseteq T_{2}$ or $T_{2} \subseteq T-T_{1}$ in which case $T_{1} \cap T_{2}=\varnothing$. If $e_{2} \in E\left(T_{1}\right)$, then $T_{2} \subseteq T_{1}$ : otherwise, $T_{1} \cap T_{2} \neq \varnothing$ and $T-T_{1} \subseteq T_{2}$, so that $w\left(T_{1}\right)+w\left(T_{2}\right)>w(T)$, contrary to the assumption.

Observation 3.2. Let $(T, w)$ be a weighted tree such that every leaf has weight 1 . Assume that we know the total weight $w(T)$ of $T$ and that, for each $j \leqslant w(T) / 2$ and each $j$-form $F$, we know the number of shapes of $(T, w)$ that belong to $F$. Then we know $T$.

Proof. We use Observation 3.1. We order the shapes of $(T, w)$ of weight at most $w(T) / 2$ decreasingly according to their weights. Let $m$ be the maximum weight of such a shape of $T$ and let $S_{1}, \ldots, S_{a}$ be the shapes with weight $m$. Note that we know precisely these $a$ trees. In addition, either the shapes $S_{1}, \ldots, S_{a}$ are joined in $T$ to the same vertex, or $a=2$ and $m=w(T) / 2$. In the latter case $(m=w(T) / 2)$ we know that $T$ consists of the two weighted rooted trees $S_{1}$ and $S_{2}$ (each of weight $m$ ) with an edge between their roots: this ends the proof for this case. Assume that $m<w(T) / 2$. We let $r$ be the additional vertex to which we link each of $S_{1}, \ldots, S_{a}$.

We show by descending induction on $j \in\{2, \ldots, m\}$ that we know the subtree of $T$ induced by all shapes of $T$ with weight in $\{j, \ldots,\lfloor W(T) / 2\rfloor\}$. The induction has thus been initialized above, so assume that $j \leqslant m-1$. Let $S_{1}, \ldots, S_{t}$ be the shapes of $T$ with weight in $\{j+1, \ldots,\lfloor W(T) / 2\rfloor\}$. Note that we know, in particular, each of these $t$ trees. The shapes of $T$ of weight equal to $j$, if any, are either shapes of $S_{1}, \ldots, S_{t}$ or joined to $r$ by an edge from their root. Fix a $j$-form $F$. Since we do know the total number of shapes belonging to $F$ and contained in each of $S_{1}, \ldots, S_{t}$ (because we know precisely those subtrees), we can deduce the number of shapes that belong to $F$ and are attached to $r$. As this argument applies to all $j$-forms $F$, we infer that we know the subtree of $T$ formed by all shapes with weight contained in $\{j, \ldots,\lfloor w(T) / 2\rfloor\}$. The reconstruction of $T$ is almost finished: letting $w_{0}$ be the total weight of the tree we built so far, it only remains to add $w(T)-w_{0}$ new leaves, each joined to the vertex $r$. This concludes the proof.

Let $(T, w)$ be a weighted tree. Let $\alpha(T)=\left(\alpha_{1}, \ldots, \alpha_{n}\right)$ be the weights of the shapes of $T$, with $\alpha_{1}<\cdots<\alpha_{n}$. The definition of a shape implies that $\alpha_{1} \geqslant 2$.

We shall consider connected partitions of the tree $T$, i.e., partitions of the vertex set of $T$ into connected subsets. Later in the paper we refer to connected partitions of $T$ simply as partitions of $T$. We shall also consider the partitions of the integer $w(T)$. To distinguish between them clearly, partitions of an integer are referred to as expressions. For each partition $P$ of $T$, the weights of the parts of $T$ form an expression of $w(T)$, which we call the characteristic of $P$.

- A $j$-expression of an integer $m$ is a partition of $m$ where one of the parts is equal to $m-j$.

- For $i \in\{1, \ldots, \ell\}$, let $m_{i}$ be an integer and $E_{i}$ an expression of $m_{i}$. We define $\left[E_{1}, \ldots, E_{\ell}\right]$ to be the expression of $\sum_{i=1}^{\ell} m_{i}$ equal to the concatenation of $E_{1}, \ldots, E_{\ell}$. In particular, if $S$ is a shape of $T$ with weight $\alpha_{j}$, then $\left[\operatorname{Ex}(S), w(T)-\alpha_{j}\right]$ is an $\alpha_{j}$-expression of $w(T)$.

- A $j$-partition of $T$ is a partition of $T$ whose characteristic is a $j$-expression of $w(T)$. In other words, one of the components of the partition has weight $w(T)-j$.

- A $j$-partition $\left(T_{0}, \ldots, T_{k}\right)$ of $T$ with $w\left(T_{0}\right)=w(T)-j$ is shaped if there exists an edge $e$ of $T$ such that $T_{0}$ is one of the components of $T-e$. Any such edge $e$ is then associated to $\left(T_{0}, \ldots, T_{k}\right)$.

- If $S$ is a shape of $T$ with weight $\alpha_{j}$ and vertex set $V(S)=\left\{v_{1}, \ldots, v_{s}\right\}$, we define $P(S)$ to be $\left(V(T) \backslash V(S),\left\{v_{1}\right\}, \ldots,\left\{v_{s}\right\}\right)$, which is a shaped $\alpha_{j}$-partition of $T$. 
For an expression $E$ of a positive integer, we let $\theta(T, w, E)$ be the number of partitions of $(T, w)$ with characteristic $E$. Note that this number is 0 if $E$ is not an expression of $w(T)$. We note that there is a bijection between connected partitions and edge subsets given by taking all edges of $T$ joining two vertices in different blocks of the connected partition and thence $\theta(T, w, E)$ turns out to be the coefficient of $x_{E}$ in the W-polynomial of $(T, w)$.

We note that among the partitions of $T$ corresponding to a given expression, some are shaped and others are not. If all the vertex weights are equal to one, we abbreviate $\theta(T, w, E)$ as $\theta(T, E)$. The proofs of both theorems rely on the following procedure.

\section{Procedure 1.}

INPUT: The polynomial $W_{(T, w)}$; an integer $j \in\left\{\alpha_{2}, \ldots, \alpha_{\ell}\right\}$, where $\ell$ is the least integer $i$ such that $\alpha_{i}>w(T) / 2$; a $j$-expression $E$ of $w(T)$ and, for each $j^{\prime}<j$ and each $j^{\prime}$-form $F$, the number of shapes $S$ of $T$ that are isomorphic to a member of $F$ (hence, according to the notation introduced above, possibly but not necessarily r-isomorphic, and hence not necessarily a member of $F$ ). OUTPUT: The number of shaped $j$-partitions of $T$ with characteristic $[w(T)-j, E]$.

Let us see how this procedure allows us to establish Theorem 1 .

Proof of Theorem 1. Fix two good weighted trees $(T, w)$ and $\left(T^{\prime}, w^{\prime}\right)$ with $W_{(T, w)}=W_{\left(T^{\prime}, w^{\prime}\right)}$. By Observation 3.2 $(T, w)$ and $\left(T^{\prime}, w^{\prime}\right)$ are isomorphic if $w(T)=w^{\prime}\left(T^{\prime}\right)$ and for each $j$-form $F$ where $j \leqslant w(T) / 2$, the numbers of shapes of $T$ and of $T^{\prime}$ that belong to $F$ are equal. To establish this, first note that the vector $\alpha(T)=\left(\alpha_{1}, \ldots, \alpha_{n}\right)$ can be computed from $W_{(T, w)}$, since the coordinates correspond to the partitions of $T$ into two subtrees (each with at least two vertices). Thus $\alpha\left(T^{\prime}\right)=\alpha(T)$.

We prove by induction on $j \in\left\{\alpha_{1}, \ldots,\lfloor w(T) / 2\rfloor\right\}$ that for every $j$-form $F$, the numbers of shapes of $T$ and of $T^{\prime}$ that belong to $F$ are the same. So suppose first, as the base case of the induction, that $j=\alpha_{1}$. Recall that $\alpha_{1} \geqslant 2$. Furthermore, a shape $S$ of $T$ or $T^{\prime}$ belongs to an $\alpha_{1}$-form if and only if $S$ is the star on $\alpha_{1}$ vertices rooted at its centre. This is because the leaves and their neighbours have weight 1 . It follows that the number of shapes of $T$ of weight $\alpha_{1}$ can be calculated from $W_{(T, w)}$ and thus this number is the same for $\left(T^{\prime}, w^{\prime}\right)$.

Now we establish the induction step. For convenience, if $F$ is a $j$-form, let $n_{T}(F)$ be the number of shapes of $T$ that belong to $F$; we use a similar notation for $T^{\prime}$. Let $j \in\left\{\alpha_{1}+1, \ldots,\lfloor w(T) / 2\rfloor\right\}$. The induction hypothesis is that $n_{T}\left(F^{\prime}\right)=n_{T^{\prime}}\left(F^{\prime}\right)$ for every $j^{\prime}$-form $F^{\prime}$ and every $j^{\prime}<j$. We want to establish that

$$
\text { for every } j \text {-form } F, \quad n_{T}(F)=n_{T^{\prime}}(F) \text {. }
$$

This will prove Theorem 1 by Observation 3.2 .

We first set a partial order on the $j$-forms, which allows us to link tree partitions with $j$-forms. Given a $j$-form $F$, we define $\operatorname{Ex}(F)$ to be $\operatorname{Ex}(f)$ for an arbitrary representative $f$ of $F$. (This definition is valid, since all representatives of a $j$-form are r-isomorphic rooted weighted trees.) A $j$-form $F^{\prime}$ is smaller than a $j$-form $F$ if $\operatorname{Ex}\left(F^{\prime}\right)$ is a proper refinement of $\operatorname{Ex}(F)$. If $P=\left(T_{0}, \ldots, T_{k}\right)$ is a shaped $j$-partition of $T$ where $w\left(T_{0}\right)=w(T)-j$, we define $S(P)$ to be the shape of $T$ formed by the union of all parts of $T$ different from $T_{0}$, that is, $S(P):=\cup_{i=1}^{k} T_{i}=T-T_{0}$, rooted at the end-vertex of the edge associated to $P$.

A key observation is that if $P$ is a shaped $j$-partition of $T$ with characteristic $[\operatorname{Ex}(F), w(T)-j]$ for some $j$-form $F$, then $\operatorname{Ex}(S(P))$ is a refinement of $\operatorname{Ex}(F)$, possibly equal to $\operatorname{Ex}(F)$.

We prove (3.1) by induction on the $j$-form $F$ considered (with respect to the partial order defined above).

We first deal with the case where $T$ has no shape that belongs to a $j$-form $F^{\prime}$ such that $\operatorname{Ex}\left(F^{\prime}\right)$ is a proper refinement of $\operatorname{Ex}(F)$. We demonstrate the following assertion. 
Assertion 3.3. If $T$ has no $j$-form $F^{\prime}$ such that $\operatorname{Ex}\left(F^{\prime}\right)$ is a proper refinement of $\operatorname{Ex}(F)$, then the number of shaped $j$-partitions of $T$ with characteristic $[\operatorname{Ex}(F), w(T)-j]$ is equal to $n_{T}(F)$.

This assertion implies that $n_{T}(F)=n_{T^{\prime}}(F)$ since by Procedure 1 and by the induction hypothesis, the number of shaped $j$-partitions of $T$ with characteristic $[\operatorname{Ex}(F), w(T)-j]$ is equal to the number of shaped $j$-partitions of $T^{\prime}$ with characteristic $[\operatorname{Ex}(F), w(T)-j]$.

To establish Assertion 3.3, we first note that each shape of $T$ that belongs to $F$ provides exactly one shaped $j$-partition of $T$ with characteristic $[\operatorname{Ex}(F), w(T)-j]$. On the other hand, if $P$ is a shaped $j$-partition of $T$ with characteristic $[\operatorname{Ex}(F), w(T)-j]$, then $\operatorname{Ex}(S(P))$ is a refinement of $\operatorname{Ex}(F)$, which by our hypothesis on $F$ must be equal to $\operatorname{Ex}(F)$. So $S(P)$ gives rise to precisely one shaped $j$-partition of $T$ with characteristic $[\operatorname{Ex}(F), w(T)-j]$, namely $P$. As $\operatorname{Ex}(F)=\operatorname{Ex}(S(P))$, it follows from Definition 2.1 $(3)$ that $S(P)$ belongs to $F$, which ends the proof of Assertion 3.3 .

In the induction step we assume that $n_{T}\left(F^{\prime}\right)=n_{T^{\prime}}\left(F^{\prime}\right)$ for every $j$-form $F^{\prime}$ such that $\operatorname{Ex}\left(F^{\prime}\right)$ is a proper refinement of $\operatorname{Ex}(F)$. Observe that for each $j$-form $F^{\prime}$ with $F^{\prime}<F$, each shape of $T$ that belongs to $F^{\prime}$ gives rise to a certain number of shaped $j$-partitions of $T$ with characteristic $\operatorname{Ex}(F)$, and this number depends only on $F^{\prime}$. Thus the number $n_{T}^{\prime}(F)$ of shaped $j$-partitions of $T$ with characteristic $[\operatorname{Ex}(F), w(T)-j]$ such that $\operatorname{Ex}(S(P))$ is a proper refinement of $\operatorname{Ex}(F)$ depends only on the multi-set $\left\{n_{T}\left(F^{\prime}\right): F^{\prime}<F\right\}$. As $\left\{n_{T}\left(F^{\prime}\right): F^{\prime}<F\right\}=\left\{n_{T^{\prime}}\left(F^{\prime}\right): F^{\prime}<F\right\}$, we deduce that $n_{T}^{\prime}(F)=n_{T^{\prime}}^{\prime}(F)$. We demonstrate the following assertion.

Assertion 3.4. The number of shaped $j$-partitions of $T$ with characteristic $[\operatorname{Ex}(F), w(T)-j]$ is equal to $n_{T}^{\prime}(F)+n_{T}(F)$.

This assertion follows analogously as Assertion 3.3. Moreover, we established in the paragraph above that $n^{\prime}(T, F)=n^{\prime}\left(T^{\prime}, F\right)$. Since the number of shaped partitions of $T$ with characteristic $[\operatorname{Ex}(F), w(T)-j]$ is equal to the number of shaped partitions of $T^{\prime}$ with characteristic $[\operatorname{Ex}(F), w(T)-j]$ by Procedure 1, we deduce that $n_{T}(F)=n_{T^{\prime}}(F)$ by Assertion 3.4. This establishes (3.1), and hence finishes the proof of Theorem 1

As we see next, the notion of a shape and Procedure 1 turn out to be essential tools to study Stanley's isomorphism conjecture restricted to caterpillars.

\section{Caterpillars}

We first observe that Theorem 2 is true for all caterpillars with at most two vertices. Hence we will assume that a caterpillar has at least three vertices in this section, and we only consider weights to be 1 ; since there is then no risk of confusion, we abbreviate $|V(T)|$ to $|T|$ for every tree $T$. Let $T$ be a caterpillar (with at least three vertices). The spine of $T$ is the unique path $P$ of $T$ such that every leaf of $T$ is at distance exactly one from a vertex of $P$.

Before proving Theorem 2, we formalize a simple but crucial observation, which is used repeatedly and implicitly in the proof of Theorem 2 .

Observation 4.1. Every shape of a caterpillar $T$ is rooted at a vertex of the spine of $T$.

It follows from Observation 4.1 that for every integer $j$, the number of shapes of $T$ with $j$ vertices belongs to $\{0,1,2\}$.

If $T$ is a caterpillar, and $E$ is an expression of $j$ so that no part of $E$ is equal to $|T|-j$, then we define $\theta_{s}(T, E)$ to be the number of shaped $j$-partitions of $T$ with characteristic $[|T|-j, E]$. Let $S_{k}$ be the star on $k$ vertices - thus $S_{1}$ is a single vertex. We always consider a star to be rooted at its center. If $T$ is a rooted tree then we define $S_{k} \rightarrow T$ to be the tree rooted at the center of $S_{k}$ and obtained by joining the root of $T$ to that of $S_{k}$ by an edge. Hence if $T$ is a rooted caterpillar, then $S_{k} \rightarrow T$ is also a rooted caterpillar. 
Let $\mathcal{A}$ be the collection of rooted caterpillars $A$ such that

- $A$ is a single vertex; or

- $A$ is a rooted edge; or

- $|A| \geqslant 3$ and the root of $A$ is either an end-vertex of the spine or a leaf attached to an end-vertex of the spine.

If $A \in \mathcal{A}$ then the reverse $\tilde{A}$ of $A$ is defined as follows. If $A$ is a single vertex then $\tilde{A}:=A$. If $A$ is a rooted edge then $\tilde{A}$ is the same edge rooted at the other end-vertex. If $A$ has at least three vertices and the root is an end-vertex of the spine then $\tilde{A}$ is obtained from $A$ by resetting the root at the other end-vertex of the spine. If $A$ has at least three vertices and the root is a leaf attached to an end-vertex of the spine then $\tilde{A}$ is obtained from $A$ by resetting the root at an arbitrary leaf attached to the other end-vertex of the spine. (We note that such a leaf always exists by the definition of the spine.)

Observation 4.2. Let $A, B \in \mathcal{A}$ such that $A$ and $B$ are isomorphic but not $r$-isomorphic. Let o, $o_{1}$ and $o_{2}$ be positive integers.

(1) The caterpillars $S_{o} \rightarrow A$ and $S_{o} \rightarrow B$ are not isomorphic; and

(2) neither are the caterpillars $S_{O_{2}} \rightarrow S_{O_{1}} \rightarrow A$ and $S_{O_{2}} \rightarrow S_{O_{1}} \rightarrow B$.

Proof. The statements are vacuously true if $|A| \leqslant 2$, so we assume that $A$ has at least three vertices - and thus so has $B$. Given an element $C \in \mathcal{A}$ with $|C| \geqslant 3$, we let $r_{C}$ be the root of $C$ and we define the degree sequence $s_{C}$ of $C$ as follows. Let $w_{1} \ldots w_{t}$ be the spine of $C$, where $w_{1}$ is closest to $r_{C}$. The degree sequence of $C$ is $s_{C}:=\left(\operatorname{deg}\left(w_{1}\right), \ldots, \operatorname{deg}\left(w_{t}\right)\right)$. The reverse of $s_{C}$ is then the sequence $\left(\operatorname{deg}\left(w_{t}\right), \ldots, \operatorname{deg}\left(w_{1}\right)\right)$. We observe that two elements $C$ and $C^{\prime}$ of $\mathcal{A}$ (with at least three vertices) are isomorphic if and only if $s_{C}=s_{C^{\prime}}$ or $s_{C^{\prime}}$ is the reverse of $s_{C}$. Furthermore, $C$ and $C^{\prime}$ are r-isomorphic if and only if $s_{C}=s_{C^{\prime}}$ and $\operatorname{deg}\left(r_{C}\right)=\operatorname{deg}\left(r_{C^{\prime}}\right)$ (that is, either both roots have degree one, or both roots have degree greater than one).

Let us make another preliminary remark. If $\operatorname{deg}_{A}\left(r_{A}\right)=1 \neq \operatorname{deg}_{B}\left(r_{B}\right)$, then in each of (1) and (2) the caterpillars obtained from $A$ and from $B$ have spines of different lengths, so they are not isomorphic. We can thus assume that either both of $r_{A}$ and $r_{B}$ have degree one, or both have degree greater than one. This implies that $s_{A} \neq s_{B}$ and $t>1$, as otherwise $A$ and $B$ would be r-isomorphic. Consequently, $s_{B}$ is the reverse of $s_{A}$. Let us write $s_{A}=\left(a_{1}, \ldots, a_{t}\right)$.

(1). For convenience, set $A^{\prime}:=S_{o} \rightarrow A$ and $B^{\prime}:=S_{o} \rightarrow B$. We know that $s_{B}=\left(a_{t}, \ldots, a_{1}\right) \neq$ $s_{A}$. Suppose first that $\operatorname{deg}_{A}\left(r_{A}\right)=1=\operatorname{deg}_{B}\left(r_{B}\right)$. Then $s_{A^{\prime}}=\left(o, 2, a_{1}, \ldots, a_{t}\right)$ if $o>1$ while $s_{A^{\prime}}=\left(2, a_{1}, \ldots, a_{t}\right)$ if $o=1$. Similarly, $s_{B^{\prime}}=\left(o, 2, a_{t}, \ldots, a_{1}\right)$ if $o>1$ while $s_{B^{\prime}}=\left(2, a_{t}, \ldots, a_{1}\right)$ if $o=1$. In either case, we see that $s_{A^{\prime}} \neq s_{B^{\prime}}$ as $s_{A} \neq s_{B}$. So suppose for a contradiction that $s_{B^{\prime}}$ is the reverse of $s_{A^{\prime}}$. In the former case, i.e., $o>1$, this means that $\left(o, 2, a_{1}, \ldots, a_{t}\right)=$ $\left(a_{1}, \ldots, a_{t}, 2, o\right)$. Then $a_{j}=o$ for $j$ odd and $a_{j}=2$ for $j$ even. In addition, $a_{t}=o$ and $a_{t-1}=2$, showing that $t$ must be odd unless $o=2$. However, either way this yields that $s_{A}=s_{B}$, a contradiction. In the latter case, i.e., $o=1$, we have $\left(2, a_{1}, \ldots, a_{t}\right)=\left(a_{1}, \ldots, a_{t}, 2\right)$, so $a_{i}=2$ for each $i \in\{1, \ldots, t\}$ which again contradicts that $s_{A} \neq s_{B}$.

It remains to deal with the case where $\operatorname{deg}_{A}\left(r_{A}\right) \neq 1 \neq \operatorname{deg}_{B}\left(r_{B}\right)$. If $o>1$, then $s_{A^{\prime}}=$ $\left(o, 1+a_{1}, a_{2}, \ldots, a_{t}\right)$ and $s_{B^{\prime}}=\left(o, 1+a_{t}, a_{t-1}, \ldots, a_{1}\right)$. If $o=1$, then $s_{A^{\prime}}=\left(1+a_{1}, a_{2}, \ldots, a_{t}\right)$ and $s_{B^{\prime}}=\left(1+a_{t}, a_{t-1}, \ldots, a_{1}\right)$. In either case, note that $s_{A^{\prime}} \neq s_{B^{\prime}}$ because $s_{A} \neq s_{B}$. Further, if $s_{B^{\prime}}$ is the reverse of $s_{A^{\prime}}$, then it implies that $o>1, a_{t}=o=a_{1}$ and $a_{i}=o+1$ for $i \in\{2, \ldots, t-1\}$, leading to $s_{A}=s_{B}$, a contradiction. This ends the proof of (1). 
(2). For convenience, set $A^{\prime}:=S_{o_{2}} \rightarrow S_{o_{1}} \rightarrow A$ and $B^{\prime}:=S_{o_{2}} \rightarrow S_{o_{1}} \rightarrow B$. Assume first that $\operatorname{deg}_{A}\left(r_{A}\right)=1=\operatorname{deg}_{B}\left(r_{B}\right)$. Then we infer as before that

$$
s_{A^{\prime}}= \begin{cases}\left(2,2, a_{1}, \ldots, a_{t}\right) & \text { if } o_{1}=1 \text { and } o_{2}=1, \\ \left(o_{2}, 2,2, a_{1}, \ldots, a_{t}\right) & \text { if } o_{1}=1 \text { and } o_{2}>1 \\ \left(1+o_{1}, 2, a_{1}, \ldots, a_{t}\right) & \text { if } o_{1}>1 \text { and } o_{2}=1 \\ \left(o_{2}, 1+o_{1}, 2, a_{1}, \ldots, a_{t}\right) & \text { if } o_{1}>1 \text { and } o_{2}>1\end{cases}
$$

and

$$
s_{B^{\prime}}= \begin{cases}\left(2,2, a_{t}, \ldots, a_{1}\right) & \text { if } o_{1}=1 \text { and } o_{2}=1 \\ \left(o_{2}, 2,2, a_{t}, \ldots, a_{1}\right) & \text { if } o_{1}=1 \text { and } o_{2}>1 \\ \left(1+o_{1}, 2, a_{t}, \ldots, a_{1}\right) & \text { if } o_{1}>1 \text { and } o_{2}=1 \\ \left(o_{2}, 1+o_{1}, 2, a_{t}, \ldots, a_{1}\right) & \text { if } o_{1}>1 \text { and } o_{2}>1\end{cases}
$$

We see that in each of the four possible cases $s_{A^{\prime}} \neq s_{B^{\prime}}$ as $s_{A} \neq s_{B}$. In addition, in none of these fours cases can $s_{B^{\prime}}$ be the reverse of $s_{A^{\prime}}$, showing that $A^{\prime}$ and $B^{\prime}$ are not isomorphic. For instance, in the second case it would imply that $t$ is 1 modulo 3 and $a_{i}=o_{2}$ if $i$ is equal to 1 modulo 3 , while $a_{i}=2$ otherwise; however this would yield that $s_{A}=s_{B}$, a contradiction. To check the fourth case, it is useful to consider the value of $t$ modulo 3 .

It remains to deal with the case where $\operatorname{deg}_{A}\left(r_{A}\right) \neq 1 \neq \operatorname{deg}_{B}\left(r_{B}\right)$. We infer the following expressions.

$$
s_{A^{\prime}}= \begin{cases}\left(2,1+a_{1}, a_{2}, \ldots, a_{t}\right) & \text { if } o_{1}=1 \text { and } o_{2}=1 \\ \left(o_{2}, 2,1+a_{1}, a_{2}, \ldots, a_{t}\right) & \text { if } o_{1}=1 \text { and } o_{2}>1 \\ \left(1+o_{1}, 1+a_{1}, a_{2}, \ldots, a_{t}\right) & \text { if } o_{1}>1 \text { and } o_{2}=1 \\ \left(o_{2}, 1+o_{1}, 1+a_{1}, a_{2}, \ldots, a_{t}\right) & \text { if } o_{1}>1 \text { and } o_{2}>1\end{cases}
$$

and

$$
s_{B^{\prime}}= \begin{cases}\left(2,1+a_{t}, a_{t-1}, \ldots, a_{1}\right) & \text { if } o_{1}=1 \text { and } o_{2}=1 \\ \left(o_{2}, 2,1+a_{t}, a_{t-1}, \ldots, a_{1}\right) & \text { if } o_{1}=1 \text { and } o_{2}>1 \\ \left(1+o_{1}, 1+a_{t}, a_{t-1}, \ldots, a_{1}\right) & \text { if } o_{1}>1 \text { and } o_{2}=1 \\ \left(o_{2}, 1+o_{1}, 1+a_{t}, a_{t-1}, \ldots, a_{1}\right) & \text { if } o_{1}>1 \text { and } o_{2}>1\end{cases}
$$

It follows that in none of the four cases the sequence $s_{B^{\prime}}$ ie equal to $s_{A^{\prime}}$ or to the reverse of $s_{A^{\prime}}$, again relying on the fact that $s_{A} \neq s_{B}$.

We are now ready to proceed with the proof of Theorem 2 .

Proof of Theorem 2. Let $T$ be a caterpillar. We proceed by induction on the number of vertices of $T$, the theorem being true if $|T|<4$. We now deal with the inductive step. As before, we note that the vector $\alpha(T)=\left(\alpha_{1}, \ldots, \alpha_{n}\right)$ can be computed from $U_{T}$, since the coordinates correspond to the partitions of $T$ into two subtrees (each with at least two vertices). We prove by induction on $j \in\left\{\alpha_{1}, \ldots,\lfloor|T| / 2\rfloor\right\}$ that for every $j$-form $F$, we can deduce from $U_{T}$ the number of shapes of $T$ that belong to $F$. Observation 3.2 ensures then that we can reconstruct $T$. Analogously as in a previous proof the number of shapes of $T$ of size $\alpha_{1}$ can be calculated from $U_{T}$. This number is one or two since $T$ is a caterpillar.

We proceed inductively and, at each step of the inductive process, we update our knowledge of the two ends of $T$, by increasing the size of our knowledge of (at least) one end of $T$. It is important to note that to know the number of shapes of $T$ that belong to a given $j$-form $F$ for some $j \geqslant 2$, it is enough to know both ends of $T$ of order $j$. At any given step, we let $R_{1}$ and $R_{2}$ be the currently known forms of the two ends of $T$. Hence after the first step $R_{1}=S_{\alpha_{1}}$ and 
$R_{2}=\varnothing$ or $R_{2}=R_{1}$, depending on whether $\theta\left(T,\left[|T|-\alpha_{1}, \alpha_{1}\right]\right)$ equals 1 or 2 . (As reported earlier, this number can be deduced from the $U$-polynomial of $T$.)

Let $j \in\left\{\alpha_{1}+1, \ldots,\lfloor|T| / 2\rfloor\right\}$. We assume that for each $j^{\prime} \in\left\{\alpha_{1}, \ldots, j-1\right\}$ and each $j^{\prime}$-form $F$ we know the number of shapes of $T$ that belong to $F$. Let us establish this last statement for $j^{\prime}=j$. If $j \notin\left\{\alpha_{2}, \ldots, \alpha_{n}\right\}$, then we know that the sought number is 0 , by the definition of $\left(\alpha_{1}, \ldots, \alpha_{n}\right)$. So we suppose now that $j=\alpha_{k}$ for some integer $k \in\{2, \ldots, n\}$. We set $m:=\alpha_{k}-\alpha_{k-1}$. (Recall that this number can be deduced from the $U$-polynomial.) Let $\alpha_{k-1}=\left|R_{1}\right| \geqslant\left|R_{2}\right|$, with $R_{2}$ possibly empty. Set $p:=\alpha_{k}-\left|R_{2}\right|$, let $R_{1}^{\prime}:=S_{m} \rightarrow R_{1}$ and $R_{2}^{\prime}:=S_{p} \rightarrow R_{2}$.

If $R_{1}$ and $R_{2}$ are r-isomorphic and $\alpha_{k}=1$ then we set $R_{1}:=R_{1}^{\prime}$ and leave $R_{2}$ unchanged. If $R_{1}$ and $R_{2}$ are r-isomorphic and $\alpha_{k}=2$ then we set $R_{1}:=R_{1}^{\prime}$ and $R_{2}:=R_{2}^{\prime}$. Hence from now on we assume that $R_{1}$ and $R_{2}$ are not r-isomorphic. We distinguish three cases.

[(1)] Let $T$ have two $\alpha_{k}$-shapes.

Then we update both $R_{1}$ and $R_{2}$, that is, we set $R_{1}:=R_{1}^{\prime}$ and $R_{2}:=R_{2}^{\prime}$.

[(2)] Let $T$ have exactly one $\alpha_{k}$-shape, i.e., either $R_{1}^{\prime}$ or $R_{2}^{\prime}$. Moreover let $R_{1}^{\prime}$ and $R_{2}^{\prime}$ be not isomorphic.

We recall that $\alpha_{k} \leqslant|T| / 2$. As $\left|R_{i}^{\prime}\right|<|T|$, we know by induction that $U_{R_{1}^{\prime}} \neq U_{R_{2}^{\prime}}$. Hence there is an expression $E^{\prime}$ of $\left|R_{1}^{\prime}\right|=\alpha_{k}$ such that $r_{1}:=\theta\left(R_{1}^{\prime}, E^{\prime}\right) \neq r_{2}:=\theta\left(R_{2}^{\prime}, E^{\prime}\right)$.

Now comes an important observation that will be used repeatedly in this proof: we know there is only one $\alpha_{k}$-shape in $T$, and thus all shaped $\alpha_{k}$-partitions of $T$ have to come from partitions where one removes the edge associated to this shape and any subset of edges inside this shape.

Therefore, there is a unique $i \in\{1,2\}$ such that $\theta_{s}\left(T, E^{\prime}\right)=r_{i}$ and we can determine it by Procedure 1. We set $R_{i}:=R_{i}^{\prime}$ and leave $R_{3-i}$ unchanged.

[(3)] Let $T$ have exactly one $\alpha_{k}$-shape, i.e., either $R_{1}^{\prime}$ or $R_{2}^{\prime}$. Moreover let $R_{1}^{\prime}$ and $R_{2}^{\prime}$ be isomorphic but not $r$-isomorphic.

In this case we explicitly know the unique isomorphism class for the $\alpha_{k}$-shapes of $T$. Therefore we know, for each $\alpha_{k}$-form $F$, the number of shapes of $T$ that are isomorphic (but not necessarily r-isomorphic) to a member of $F$. We observe that $k<n$. We set $q:=\alpha_{k+1}-\alpha_{k}$.

By Procedure 1, we know for each $\alpha_{k+1}$-expression $E$ the number of shaped $\alpha_{k+1}$-partitions of $T$ with characteristic $E$.

There are four candidates for an $\alpha_{k+1}$-shape of $T$, namely $S_{1,1}:=S_{q} \rightarrow S_{m} \rightarrow R_{1}=S_{q} \rightarrow R_{1}^{\prime}$, $S_{2,1}:=S_{q+m} \rightarrow R_{1}, S_{1,2}:=S_{q+p} \rightarrow R_{2}$ and $S_{2,2}:=S_{q} \rightarrow S_{p} \rightarrow R_{2}=S_{q} \rightarrow R_{2}^{\prime}$.

We now introduce some labels for the vertices of the stars $S_{q}, S_{q+m}$ and $S_{q+p}$. The vertices of $S_{q}$ are labelled $v_{1}, \ldots, v_{q}$, where $v_{q}$ is the centre of $S_{q}$. By extension, the corresponding vertices of $S_{1,1}$ and $S_{2,2}$ inherit those labels. For $i \in\{p, m\}$, the vertices of $S_{q+i}$ are labelled $v_{1}, \ldots, v_{q+i}$ where, this time, the labels $v_{1}, \ldots, v_{q}$ are assigned to leaves only. Similarly, the corresponding vertices of $S_{1,2}$ and of $S_{2,1}$ inherit those labels. Thus, for example, the vertex $v_{q}$ of $S_{1,1}$ is the centre of the star $S_{q}$ and hence the root of $S_{1,1}$, while the vertex $v_{q}$ of $S_{1,2}$ is one of the leaves of $S_{q+p}$ and hence is adjacent to the root of $S_{1,2}$.

[(3.1)] Let $T$ have two $\alpha_{k+1}$-shapes.

There are two possibilities for the two $\alpha_{k+1}$-shapes of $T$ : either $S_{1,1}, S_{1,2}$ or $S_{2,1}, S_{2,2}$. We note that this implies that $\alpha_{k+1} \leqslant|T| / 2$. For $i \in\{1,2\}$, let $T_{i}$ be any caterpillar with $\left|T_{i}\right|=|T|$ whose $\alpha_{k+1}$-shapes are exactly $S_{i, 1}$ and $S_{i, 2}$.

Observation 4.3. If $q>1$ then $S_{i, j}$ and $S_{i^{\prime}, j^{\prime}}$ are not isomorphic (as unrooted trees) whenever $i, i^{\prime}, j, j^{\prime} \in\{1,2\}$ with $i \neq i^{\prime}$.

Proof. Comparing the lengths of the spines, the only possible pairs of isomorphic trees are: $S_{1,1}$ with $S_{2,2}$, and $S_{1,2}$ with $S_{2,1}$. However, the fact that $R_{1}^{\prime}$ and $R_{2}^{\prime}$ are isomorphic but not 
r-isomorphic prevents each of these pairs to consist of isomorphic trees, using Observation 4.2 1 . for the former one.

Let $E$ be an expression of $\alpha_{k+1}$. We note that each $T_{i}$ has exactly two vertices labelled by $v_{q}$, namely the root of $S_{i, i}$ and a leaf of $S_{i, 3-i}$ attached to the root of $S_{i, 3-i}$. We classify the shaped $\alpha_{k+1}$-partitions of $T_{i}$, for each $i \in\{1,2\}$, into four classes $C(E, i, 1), C(E, i, 2), C(E, i, 3)$ and $C(E, i, 4)$. To this end, let $\mathcal{E}_{i}$ be the collections of all shaped $\alpha_{k+1}$-partitions of $T_{i}$ of characteristic $\left[|T|-\alpha_{k+1}, E\right]$. An element $P$ of $\mathcal{E}_{i}$ partitions a subtree $G$ of $T_{i}$ if a subset (possibly of order one) of the parts of $P$ forms a partition of $G$.

(1) We let $C(E, i, 1)$ be the collection of all elements of $\mathcal{E}_{i}$ such that a subset of parts of $P$ is a partition of the unique $\alpha_{k}$-shape of $T_{i}$.

(2) We let $C(E, i, 2)$ be the collection of all elements $P \in \mathcal{E}_{i} \backslash C(E, i, 1)$ such that $\left\{v_{q}\right\} \subset$ $V\left(S_{i, i}\right) \cup V\left(S_{i, 3-i}\right)$ is not a part of $P$.

(3) We let $C(E, i, 3)$ be the collection of all elements $P \in \mathcal{E}_{i} \backslash C(E, i, 1)$ such that $\left\{v_{i}\right\} \subseteq$ $V\left(S_{i, 3-i}\right)$ is a part of $P$ for each $i \leqslant q$.

(4) We let $C(E, i, 4)$ be the collection of all elements $P \in \mathcal{E}_{i} \backslash C(E, i, 1)$ such that $\left\{v_{q}\right\} \subseteq$ $V\left(S_{i, 3-i}\right)$ is a part of $P$ and there exists $\ell \in\{1, \ldots, q-1\}$ such that $\left\{v_{\ell}\right\} \subseteq V\left(S_{i, 3-i}\right)$ is not a part of $P$.

Observation 4.4. Let $i \in\{1,2\}$ and let $E$ be an expression of $\alpha_{k+1}$.

(1) The partitions in $C(E, i, 1)$ partition the shape $S_{i, i}$ of $T_{i}$. Moreover, there is a bijection $F$ from $C(E, 1,1)$ to $C(E, 2,1)$ so that for each $P$, there is a bijection between the sets of components of $P$ and $F(P)$ that identifies the class of $P$ containing the root of the $\left(\alpha_{k+1}\right)$-shape with the class of $F(P)$ containing the root of the $\left(\alpha_{k+1}\right)$-shape.

(2) There is a bijection $F$ from $C(E, 1,2)$ to $C(E, 2,2)$ so that if $P$ partitions the shape $S_{i, j}$ of $T_{i}$, then $F(P)$ partitions the shape $S_{3-i, j}$ of $T_{3-i}$ and there is a bijection between the sets of components of $P$ and $F(P)$ that identifies the class of $P$ containing the root of the $\left(\alpha_{k+1}\right)$-shape with the class of $F(P)$ containing the root of the $\left(\alpha_{k+1}\right)$-shape.

(3) The partitions in $C(E, i, 3)$ partition the shape $S_{i, 3-i}$ of $T_{i}$. Moreover, there is a bijection $F$ from $C(E, 1,3)$ to $C(E, 2,3)$ so that for each $P$, there is a bijection between the sets of components of $P$ and $F(P)$ that identifies the class of $P$ containing the root of the $\left(\alpha_{k+1}\right)$-shape with the class of $F(P)$ containing the root of the $\left(\alpha_{k+1}\right)$-shape.

(4) The partitions in $C(E, i, 4)$ partition the shape $S_{i, 3-i}$ of $T_{i}$.

Proof. Items (2) and (4) follow directly from the structure of the shapes $S_{i, j}$. Items (1) and (3) follow from the assumption that $R_{1}^{\prime}$ and $R_{2}^{\prime}$ are isomorphic.

Let $S^{2}:=S_{q+m-1} \rightarrow R_{1}$ and $S^{1}:=S_{q+p-1} \rightarrow R_{2}$. We observe that if $q>1$ then $S^{1}$ and $S^{2}$ are not isomorphic since none of the pairs $\left(R_{1}^{\prime}, R_{2}^{\prime}\right)$ and $\left(R_{1}, R_{2}\right)$ consists of r-isomorphic trees and, in addition, $R_{1}^{\prime}$ and $R_{2}^{\prime}$ are isomorphic.

Observation 4.5. Suppose that $q>1$ and let $E$ be an expression of $\alpha_{k+1}-1$ such that $r_{1}:=\theta\left(S^{1}, E\right) \neq \theta\left(S^{2}, E\right)=: r_{2}$. Such an expression $E$ exists by the induction assumption since $\alpha_{k+1}-1<|T|$. Let $i \in\{1,2\}$ such that $r_{i}>r_{3-i}$. Then $\theta_{s}\left(T_{i},[E, 1]\right)>\theta_{s}\left(T_{3-i},[E, 1]\right)$.

Proof. Let $E^{\prime}:=[E, 1]$. By Observation 4.4 it suffices to show that $\left|C\left(E^{\prime}, i, 4\right)\right|>\left|C\left(E^{\prime}, 3-i, 4\right)\right|$, which can be argued as follows.

We first observe that $\left|C\left(E^{\prime}, j, 4\right)\right|=r_{j}-\left|C\left(E^{\prime}, j, 3\right)\right|$ for each $j \in\{1,2\}$. Further, $\left|C\left(E^{\prime}, 1,3\right)\right|=$ $\left|C\left(E^{\prime}, 2,3\right)\right|$ by Observation 4.4. As we assumed that $r_{i}>r_{3-i}$, the observation holds.

[(3.1.1)] Let $q>1$. Let $E$ be the expression from Observation 4.5. We recall that by Procedure 1, we know for each $\alpha_{k+1}$-expression $E$ the number of shaped $\alpha_{k+1}$-partitions of $T$ with 
characteristic $E$. Hence we know $\theta_{s}(T,[E, 1])$ and also $\theta_{s}(T,[E, 1]) \in\left\{\theta_{s}\left(T_{1},[E, 1]\right), \theta_{s}\left(T_{2},[E, 1]\right)\right\}$, which contains two values. Hence this case is solved by Observation 4.5 .

[(3.1.2)] Let $q=1$. Then $S_{i, i}$ is isomorphic but not r-isomorphic to $S_{3-i, i}$ for each $i \in\{1,2\}$, and $S_{1,1}$ is not isomorphic to $S_{2,2}$ since $R_{1}^{\prime}$ and $R_{2}^{\prime}$ are not r-isomorphic. We observe that $k+1<n$ since $\alpha_{k+1} \leqslant|T| / 2$ and not all $\alpha_{k+1}$-shapes of $T$ are stars.

We now know all the input data of Procedure 1 for $T$ and $j=\alpha_{k+2}$ since for each $j^{\prime} \leqslant \alpha_{k+1}$ and for each $j^{\prime}$-form $F$ the number of shapes $S$ of $T_{1}$ that are isomorphic to a member of $F$ is equal to the number of shapes $S$ of $T_{2}$ that are isomorphic to a member of $F$.

Let $q^{\prime}:=\alpha_{k+2}-\alpha_{k+1}$. There are four candidates for an $\alpha_{k+2}$-shape of $T$, namely $S_{i, j}^{\prime}=S_{q^{\prime}} \rightarrow$ $S_{i, j}$ for $(i, j) \in\{1,2\}^{2}$.

Observation 4.6. The trees $S_{i, j}^{\prime}$, for $(i, j) \in\{1,2\}^{2}$, are mutually non-isomorphic.

Proof. For $S_{1,1}^{\prime}$ and $S_{2,2}^{\prime}$, this follows from Observation 4.2 . Moreover, for each $i \in\{1,2\}$, we know that $S_{i, i}^{\prime}$ is isomorphic to neither of $S_{i, 3-1}^{\prime}$ and $S_{3-i, i}$ because the lengths of the spines are different. Finally we consider $S_{1,2}^{\prime}$ and $S_{2,1}^{\prime}$. We know that the rooted caterpillar $R_{1}^{\prime}$ is the reverse of $R_{2}^{\prime}$. Recall the degree sequences of caterpillars, defined in the proof of Observation 4.2 on page 9 . Let the degree sequence $s_{R_{1}^{\prime}}$ of $R_{1}^{\prime}$ be $\left(a_{1}, \ldots, a_{n}\right)$ - we know that $a_{1}=m$. Then $s_{S_{2,1}^{\prime}}$ is the sequence $s_{2}:=\left(a_{1}, \ldots, a_{n}+1, q^{\prime}\right)$ and $s_{S_{1,2}^{\prime}}$ is the sequence $s_{1}:=\left(a_{n}, \ldots, a_{1}+1, q^{\prime}\right)$. We observe that if $s_{1}=s_{2}$ or if $s_{1}$ is the reverse of $s_{2}$, then $\left(a_{1}, \ldots, a_{n}\right)$ is equal to its reverse, which contradicts the assumption that $R_{1}^{\prime}$ and $R_{2}^{\prime}$ are not r-isomorphic.

If $T$ has a unique $\alpha_{k+2}$-shape then we can determine which one of the four mutually nonisomorphic candidates it is using the induction assumption $\left(\alpha_{k+2}<|T|\right)$ and Procedure 1 , which implies that we know for each $\alpha_{k+2}$-expression $E$ the number of shaped $\alpha_{k+2}$-partitions of $T$ with characteristic $E$. Hence, we assume that $T$ has two $\alpha_{k+2}$-shapes.

There are two possibilities for the two $\alpha_{k+2}$-shapes of $T$ : either $S_{1,1}^{\prime}, S_{1,2}^{\prime}$ or $S_{2,1}^{\prime}, S_{2,2}^{\prime}$. For $i \in$ $\{1,2\}$, let $T_{i}^{\prime}$ be any caterpillar with $\left|T_{i}^{\prime}\right|=|T|$ whose $\alpha_{k+2}$-shapes are exactly $S_{i, 1}^{\prime}$ and $S_{i, 2}^{\prime}$.

Next we proceed analogously as in case (3.1.1). Similarly as before, let us label the vertices of the shape $S_{q^{\prime}}$ of $S_{i, j}^{\prime}$ by $u_{1}, \ldots, u_{q^{\prime}}$ for each $(i, j) \in\{1,2\}^{2}$, where $u_{q^{\prime}}$ is the centre of $S_{q^{\prime}}$.

Let $i \in\{1,2\}$ and let $E$ be an expression of $\alpha_{k+2}$. We classify the shaped $\alpha_{k+2}$-partitions of $T_{i}^{\prime}$ into four classes $C^{\prime}(E, i, 1), C^{\prime}(E, i, 2), C^{\prime}(E, i, 3)$ and $C^{\prime}(E, i, 4)$. To this end, let $\mathcal{E}_{i}^{\prime}$ be the collection of all shaped $\alpha_{k+2}$-partitions of $T_{i}$ of characteristic $\left[|T|-\alpha_{k+2}, E\right]$. An element $P$ of $\mathcal{E}_{i}^{\prime}$ partitions a subtree $G$ of $T_{i}^{\prime}$ if a subset (possibly of order one) of the parts of $P$ forms a partition of $G$.

(1) We let $C^{\prime}(E, i, 1)$ be the collection of all elements of $\mathcal{E}_{i}^{\prime}$ such that a subset of parts of $P$ is a partition the unique $\alpha_{k}$-shape of $T_{i}^{\prime}$.

(2) We let $C^{\prime}(E, i, 2)$ be the collection of all elements $P \in \mathcal{E}_{i}^{\prime} \backslash C^{\prime}(E, i, 1)$ such that $\left\{v_{q}\right\} \subseteq$ $V\left(S_{i, 3-i}\right)$ is not a part of $P$.

(3) We let $C^{\prime}(E, i, 3)$ be the collection of all elements $P \in \mathcal{E}_{i}^{\prime} \backslash C^{\prime}(E, i, 1)$ such that $\left\{v_{q}\right\} \subseteq$ $V\left(S_{i, 3-i}\right)$ is a part of $P$ and $u_{q^{\prime}} \in V\left(S_{i, 3-i}^{\prime}\right)$ does not belong to the same part of $P$ as the root of $S_{i, 3-i} \subset S_{i, 3-i}^{\prime}$.

(4) We let $C^{\prime}(E, i, 4)$ be the collection of all elements $P \in \mathcal{E}_{i}^{\prime} \backslash C^{\prime}(E, i, 1)$ such that $\left\{v_{q}\right\} \subseteq$ $V\left(S_{i, 3-i}\right)$ is a part of $P$ and $u_{q^{\prime}} \in V\left(S_{i, 3-i}^{\prime}\right)$ belongs to the same part of $P$ as the root of $S_{i, 3-i} \subset$ $S_{i, 3-i}^{\prime}$.

Observation 4.7. Let $i \in\{1,2\}$ and let $E$ be an expression of $\alpha_{k+2}$.

(1) The partitions in $C^{\prime}(E, i, 1)$ partition the shape $S_{i, i}^{\prime}$. Moreover, there is a bijection $F$ from $C^{\prime}(E, 1,1)$ to $C^{\prime}(E, 2,1)$ so that for each $P$, there is a bijection between the sets 
of components of $P$ and $F(P)$ that identifies the class of $P$ containing the root of the $\left(\alpha_{k+2}\right)$-shape with the class of $F(P)$ containing the root of the $\left(\alpha_{k+2}\right)$-shape.

(2) There is a bijection $F$ from $C^{\prime}(E, 1,2)$ to $C^{\prime}(E, 2,2)$ so that if $P$ partitions the shape $S_{i, j}$ of $T_{i}$, then $F(P)$ partitions the shape $S_{3-i, j}$ of $T_{3-i}$ and there is a bijection between the sets of components of $P$ and $F(P)$ that identifies the class of $P$ containing the root of the $\left(\alpha_{k+2}\right)$-shape with the class of $F(P)$ containing the root of the $\left(\alpha_{k+2}\right)$-shape.

(3) The partitions in $C^{\prime}(E, i, 3)$ partition the shape $S_{i, 3-i}^{\prime}$. Moreover, there is a bijection $F$ from $C^{\prime}(E, 1,3)$ to $C^{\prime}(E, 2,3)$ so that for each $P$, there is a bijection between the sets of components of $P$ and $F(P)$ that identifies the class of $P$ containing the root of the $\left(\alpha_{k+2}\right)$-shape with the class of $F(P)$ containing the root of the $\left(\alpha_{k+2}\right)$-shape.

(4) The partitions in $C^{\prime}(E, i, 4)$ partition $S_{i, 3-i}^{\prime}$.

Proof. Analogously as in the proof of Observation 4.4 the Items (2) and (4) follow directly from the structure of the $S_{i, j}^{\prime}$. Items (1) and (3) follow from the assumption that $R_{1}^{\prime}$ and $R_{2}^{\prime}$ are isomorphic.

Let $Q^{1}:=S_{q^{\prime}} \rightarrow R_{2}^{\prime}$ and $Q^{2}:=S_{q^{\prime}} \rightarrow R_{1}^{\prime}$. We note that $Q^{1}$ and $Q^{2}$ are not isomorphic by Observation 4.2

Observation 4.8. Let $E$ be an expression of $\alpha_{k+2}-1$ such that $r_{1}:=\theta\left(Q^{1}, E\right) \neq \theta\left(Q^{2}, E\right)=: r_{2}$. Let $i \in\{1,2\}$ such that $r_{i}>r_{3-i}$. Then $\theta_{s}\left(T_{i}^{\prime},[E, 1]\right)>\theta_{s}\left(T_{3-i}^{\prime},[E, 1]\right)$.

Proof. Set $E^{\prime}:=[E, 1]$. By Observation 4.7 it suffices to show that $\left|C^{\prime}\left(E^{\prime}, i, 4\right)\right|>\left|C^{\prime}\left(E^{\prime}, 3-i, 4\right)\right|$, which can be argued as follows. We first observe that $\left|C^{\prime}\left(E^{\prime}, i, 4\right)\right|=r_{i}-\left|C^{\prime}\left(E^{\prime}, i, 3\right)\right|$ for each $i \in\{1,2\}$. Next, Observation 4.7 implies that $\left|C^{\prime}\left(E^{\prime}, 1,3\right)\right|=\left|C\left(E^{\prime}, 2,3\right)\right|$. Since $r_{i}>r_{3-i}$, the observation thus holds.

We recall that by Procedure 1 we know for each $\alpha_{k+2}$-expression $E$ the number of shaped $\alpha_{k+2}$-partitions of $T$ with characteristic $E$. Hence we know $\theta_{s}(T,[E, 1])$ and also $\theta_{s}(T,[E, 1]) \in$ $\left\{\theta_{s}\left(T_{1}^{\prime},[E, 1]\right), \theta_{s}\left(T_{2}^{\prime},[E, 1]\right)\right\}$, which consists of two values. Hence the case $(3.1 .2)$ is solved by Observation 4.8

[(3.2)] Let $T$ have a unique $\alpha_{k+1}$-shape.

Let $q>1$. Using Observation 4.3 , the induction assumption and Procedure 1 and considering the shaped $\alpha_{k+1}$-partitions of $T$, we can determine if the unique $\alpha_{k+1}$-shape of $T$ is in the set $\left\{S_{1,1}, S_{1,2}\right\}$ or in the set $\left\{S_{2,1}, S_{2,2}\right\}$. In the first case the unique $\alpha_{k}$-shape of $T$ is $R_{1}^{\prime}$, in the second case the unique $\alpha_{k}$-shape of $T$ is $R_{2}^{\prime}$.

So suppose that $q=1$. There are two pairs of isomorphic candidates: $S_{1,1}$ is isomorphic to $S_{2,1}$ and $S_{1,2}$ is isomorphic to $S_{2,2}$. We observe that for each pair, its two elements differ in the number of leaves different from the root. Moreover, $S_{1,1}$ and $S_{2,2}$ are not isomorphic. By considering the shaped $\alpha_{k+1}$-partitions of $T$ we can determine to which pair the unique $\alpha_{k+1}$-shape of $T$ belongs. We may assume, without loss of generality, that it belongs to $\left\{S_{1,1}, S_{2,1}\right\}$. We now show that we can determine the number of leaves of the unique $\alpha_{k+1}$-shape of $T$ different from the root and therefore determine whether the correct shape is $\left\{S_{1,1}\right.$ or $\left.S_{2,1}\right\}$.

We observe that $n \neq k+1$ since $q=1$. Since we know the isomorphism class of the unique $\alpha_{k+1}$-shape of $T$, we can determine the number of shaped $\alpha_{k+2}$-partitions of $T$ by Procedure 1.

We have

$$
\theta\left(T,|T|-\alpha_{k+1}-1, \alpha_{k+1}, 1\right)=\theta_{s}\left(T, \alpha_{k+1}, 1\right)+d\left(T, \alpha_{k+1}, 1\right),
$$

where $d\left(T, \alpha_{k+1}, 1\right)$ is equal to the number of leaves of $T$ outside of the unique $\alpha_{k+1}$-shape. The considerations above imply that we can determine $d\left(T, \alpha_{k+1}, 1\right)$. Since we know the number of leaves of $T$, we can also determine the number of leaves of the unique $\alpha_{k+1}$-shape of $T$ that are 
different from the root. Hence we can determine whether this shape is $S_{1,1}$ or $S_{1,2}$. This finishes case (3.2) and thus case (3).

This ends our updating process and the inductive step of our induction. Consequently, we established that we know, for each $j \in\left\{\alpha_{1}, \ldots,|T| / 2\right\}$ and each $j$-form $F$, the number of shapes of $T$ that belongs to $F$. Therefore Observation 3.2 ensures that we know $T$. This concludes the induction on the size of $T$ and thus the proof of Theorem 2 .

\section{Designing Procedure 1}

A $j$-situation $\sigma$ is a sequence $\left(\left(\sigma_{1}, w_{1}\right), \ldots,\left(\sigma_{t(\sigma)}, w_{t(\sigma)}\right)\right)$ of the representatives of isomorphism classes of weighted non-rooted trees such that $t(\sigma) \geqslant 2, \sum_{i=1}^{t(\sigma)} w_{i}\left(\sigma_{i}\right)=j$ and there are numbers $p(\sigma), q(0), q(1), \ldots, q(p(\sigma))$ such that

(1) $1 \leqslant p(\sigma) \leqslant t(\sigma)$ and $0=q(0)<q(1)<\cdots<q(p(\sigma))=t(\sigma)$;

(2) for each $i \in\{0, \ldots, p(\sigma)-1\}$ the weighted trees $\left(\sigma_{q(i)+s}, w_{q(i)+s}\right)$ for $s \in\{1, \ldots, q(i+$ 1) $-q(i)\}$ are the same; and

(3) if $k \notin\{q(i)+1, \ldots, q(i+1)\}$ then the weighted tree $\left(\sigma_{k}, w_{k}\right)$ is not isomorphic to $\left(\sigma_{q(i)+1} \cdot w_{q(i)+1}\right)$.

A $j$-situation $\sigma$ is said to occur in a tree $T$ if there exists a subtree $T^{\prime}$ of $T$ and $t(\sigma)$ distinct edges $e_{1}, \ldots, e_{t(\sigma)}$ with exactly one end in $V\left(T^{\prime}\right)$ such that, for each $i \in\{1, \ldots, t(\sigma)\}$, there is an isomorphism (thus preserving the weights but not necessarily the roots) between $\sigma_{i}$ and the component of $T-e_{i}$ not containing $T^{\prime}$. Note that if $\sigma$ occurs in $T$, then for each $i \in\{1, \ldots, t(\sigma)\}$ such that $\sigma_{i}$ is not a single vertex the tree $T$ has a shape isomorphic (but not necessarily r-isomorphic) to $\sigma_{i}$.

We proceed in two steps, the first one being an exhaustive listing that depends only on $j$.

Step 1. Explicitly list all $j$-situations for $j \leqslant \alpha_{l}$.

Step 2. For each $j$-situation $\sigma$ from Step 1, compute the number $m_{T}(\sigma)$ of times $\sigma$ occurs in $T$.

Before designing Step 2, we show how Steps 1 and 2 accomplish Procedure 1 . Suppose that the two steps are completed. Let $E=\left\{w(T)-j, E_{1}, \ldots, E_{k}\right\}$ be a $j$-expression of $w(T)$.

For each $j$-situation $\sigma=\left(\left(\sigma_{1}, w_{1}\right), \ldots,\left(\sigma_{t(\sigma)}, w_{t(\sigma)}\right)\right)$, let $\Psi_{\sigma}$ be the collection of all surjections from the expression $\left\{E_{1}, \ldots, E_{k}\right\}$ to $\left\{\sigma_{1}, \ldots, \sigma_{t(\sigma)}\right\}$. Two elements $f$ and $g$ of $\Psi_{\sigma}$ are equivalent if the multi-set $f^{-1}\left(\sigma_{i}\right)$ is equal to the multi-set $g^{-1}\left(\sigma_{i}\right)$ for every $i \in\{1, \ldots, t(\sigma)\}$. We consider the equivalence classes for this relation on $\Psi_{\sigma}$ and we form $\Psi_{\sigma}^{\prime}$ by arbitrarily choosing one representative in each equivalent class. We observe that the number $X$ of non-shaped $j$-partitions of $T$ with characteristic $E$ is

$$
\left[p(\sigma) ! \prod_{1 \leqslant i \leqslant p(\sigma)}(q(i)-q(i-1)) !\right]^{-1} \sum_{j \text {-situation } \sigma} m_{T}(\sigma) \sum_{f \in \Psi_{\sigma}^{\prime}} \sum_{i=1}^{t(\sigma)} \theta\left(\sigma_{i}, w_{i}, f^{-1}\left(\sigma_{i}\right)\right),
$$

where the multi-set $f^{-1}\left(\sigma_{i}\right)$ is naturally interpreted as an expression. Indeed, a non-shaped partition of $T$ with characteristic $E$ corresponds precisely to the occurrence of some $j$-situation $\sigma=$ $\left(\left(\sigma_{1}, w_{1}\right), \ldots,\left(\sigma_{t(\sigma)}, w_{t(\sigma)}\right)\right)$ where the trees $\sigma_{1} \ldots, \sigma_{t(\sigma)}$ are also partitioned (possibly trivially). Recalling that $\theta\left(\sigma_{i}, w_{i}, E^{\prime}\right)$ is zero if $E^{\prime}$ is not an expression of $w_{i}\left(\sigma_{i}\right)$, the formula (5.1) follows. Notice that (5.1) does allow us to compute $X$ when Step 1 and Step 2 are completed. Consequently, we can compute the number of shaped $j$-partitions of $T$ with characteristic $E$, which is

$$
\theta(T, w, E)-X .
$$

This accomplishes Procedure 1 .

It remains to design Step 2. To this end, we fix a $j$-situation $\sigma=\left(\left(\sigma_{1}, w_{1}\right), \ldots,\left(\sigma_{t}, w_{t}\right)\right)$. Define $\Lambda$ to be the set of all sequences $\left(T_{1}, \ldots, T_{t}\right)$ such that for each $i \in\{1, \ldots, t\}$,

- $T_{i}$ is either a shape of $T$ or a leaf; 
- $T_{i}$ is isomorphic to $\left(\sigma_{i}, w_{i}\right)$ as a weighted non-rooted tree; and

- if $k \in\{1, \ldots, t\} \backslash\{i\}$, then $T_{i}$ is not a subtree of $T_{k}$.

Observation 5.1. The number of times that $\sigma$ occurs in $T$ is equal to $|\Lambda|$.

Proof. We prove that the elements of $\Lambda$ are exactly occurrences of $\sigma$ in $T$. By the definition, each occurrence of $\sigma$ gives rise to an element of $\Lambda$.

Conversely, let $\left(T_{1}, \ldots, T_{t}\right)$ be an element of $\Lambda$. Observation 3.1 implies that the trees $T_{i}$ are mutually disjoint. For each $i \in\{1, \ldots, t\}$, let $e_{i}$ be the edge of $T$ associated to the shape $T_{i}$, that is, $e_{i}$ connects $T_{i}$ to $T-T_{i}$; and let $v_{i}$ be the endvertex of $e_{i}$ that does not belong to $T_{i}$. Note that $v_{i} \notin \cup_{k=1}^{t} T_{k}$ since no tree $T_{i}$ is a subtree of another tree $T_{k}$ and $j \leqslant \alpha_{l}<w(T)$. Set $T_{0}^{\prime}:=T$ and $T_{i}^{\prime}:=T_{i-1}^{\prime}-T_{i}$ for $i \geqslant 1$.

Observe that each of $T_{i+1}, \ldots, T_{t}$ is either a leaf or a shape of $T_{i}^{\prime}$. Hence $T_{i}^{\prime}$ is connected and contains all the vertices $v_{1}, \ldots, v_{t}$. Therefore setting $T^{\prime}:=T_{t}^{\prime}$ shows that $\left(T_{1}, \ldots, T_{t}\right)$ occurs in $T$.

Our goal is to compute $|\Lambda|$. For a weighted tree $\left(T^{\prime}, w^{\prime}\right)$, define $\Lambda_{0}\left(T^{\prime}, w^{\prime}\right)$ to be the set of all sequences $\left(T_{1}, \ldots, T_{t}\right)$ such that for each $i \in\{1, \ldots, t\}$ it holds that $T_{i}$ is either a leaf or a shape of $T^{\prime}$ that is isomorphic to $\left(\sigma_{i}, w_{i}\right)$ as a weighted non-rooted tree. Set $\Lambda_{0}:=\Lambda_{0}(T, w)$. In this notation, the weight shall be omitted when there is no risk of confusion. The advantage of $\Lambda_{0}$ is that its size can be computed. Indeed,

$$
\left|\Lambda_{0}\right|=\prod_{i=1}^{t} \sharp\left(\left(\sigma_{i}, w_{i}\right) \hookrightarrow(T, w)\right),
$$

where $\sharp\left(\left(\sigma_{i}, w_{i}\right) \hookrightarrow(T, w)\right)$ is the number of leaves or shapes of $T$ that are isomorphic to $\left(\sigma_{i}, w_{i}\right)$ as weighted non-rooted trees. This number is given in the input of Procedure 1 since $w_{i}\left(\sigma_{i}\right)<j$.

Next, we compute $|\Lambda|$ using the principle of inclusion and exclusion. Setting $I:=\{1, \ldots, t\}^{2} \backslash$ $\{(i, i): 1 \leqslant i \leqslant t\}$, we have

$$
|\Lambda|=\left|\Lambda_{0}\right|-\left|\bigcup_{(i, k) \in I} \Lambda_{(i, k)}\right|,
$$

where $\Lambda_{(i, k)}$ is the subset of $\Lambda_{0}$ composed of the elements $\left(T_{1}, \ldots, T_{t}\right)$ with $T_{i} \subseteq T_{k}$.

By the principle of inclusion-exclusion, we deduce that the output of Step 2 is equal to

$$
\left|\Lambda_{0}\right|-\sum_{\varnothing \neq J \subseteq I}(-1)^{|J|-1}\left|\bigcap_{(i, k) \in J} \Lambda_{(i, k)}\right| .
$$

It remains to compute $\left|\bigcap_{(i, k) \in J} \Lambda_{(i, k)}\right|$ for each non-empty subset $J$ of $I$. We start with an observation, which characterises the sets $J$ for which the considered intersection is not empty.

Observation 5.2. Let $J \subseteq I$. Then, $\bigcap_{(i, k) \in J} \Lambda_{(i, k)} \neq \varnothing$ if and only if for every $(i, k) \in J$, either $\sigma_{i}$ is isomorphic to $\sigma_{k}$, or $\sigma_{k}$ has a leaf or a shape that is isomorphic to $\sigma_{i}$ as a weighted non-rooted tree.

From now on, we consider an arbitrary contributing set $J$. We construct four directed graphs $A_{0}, A_{1}, A_{2}$ and $A_{3}$ that depend on $J$. Each vertex $x$ of $A_{l}$ is labeled by a subset $\ell(x)$ of $\left\{\left(\sigma_{1}, w_{1}\right), \ldots,\left(\sigma_{t}, w_{t}\right)\right\}$. These labels will have the following properties.

(1) $(\ell(x))_{x \in V\left(A_{l}\right)}$ is a partition of $\left\{\left(\sigma_{1}, w_{1}\right), \ldots,\left(\sigma_{t}, w_{t}\right)\right\}$.

(2) For each vertex $x$ of $A_{l}$, all weighted trees in $\ell(x)$ are isomorphic.

(3) $\left|\cap_{(i, k) \in J} \Lambda_{(i, k)}\right|$ is equal to the number of elements $\left(T_{1}, \ldots, T_{t}\right)$ of $\Lambda_{0}$ such that

- for each vertex $x$ of $A_{l}$, if $\left(\sigma_{i}, w_{i}\right),\left(\sigma_{k}, w_{k}\right) \in \ell(x)$ then $T_{i}=T_{k}$; and 
- for every $\operatorname{arc}(x, y)$ of $A_{l}$, if $\left(\left(\sigma_{i}, w_{i}\right),\left(\sigma_{k}, w_{k}\right)\right) \in \ell(x) \times \ell(y)$, then $T_{i} \subseteq T_{k}$.

The directed graph $A_{0}$ is obtained as follows. We start from the vertex set $\left\{z_{1}, \ldots, z_{t}\right\}$. For each $i \in\{1, \ldots, t\}$, the label $\ell\left(z_{i}\right)$ of $z_{i}$ is set to be $\left\{\left(\sigma_{i}, w_{i}\right)\right\}$. For each $(i, k) \in J$, we add an arc from $z_{i}$ to $z_{k}$. Thus $A_{0}$ satisfies properties (1)-(3). Note that $A_{0}$ may contain directed cycles, but by Observation 5.2 if $C$ is a directed cycle then all elements in $\cup_{x \in V(C)} \ell(x)$ are isomorphic.

Now, $A_{1}$ is obtained from $A_{0}$ by the following recursive operation. Let $(x, y, z)$ be a triple of vertices such that $(x, y)$ and $(x, z)$ are arcs, but neither $(y, z)$ nor $(z, y)$ are $\operatorname{arcs}$. Let $\left(\sigma_{y}, w_{y}\right) \in \ell(y)$ and $\left(\sigma_{z}, w_{z}\right) \in \ell(z)$. We add the $\operatorname{arc}(y, z)$ if $\left|V\left(\sigma_{y}\right)\right| \leqslant\left|V\left(\sigma_{z}\right)\right|$, and the arc $(z, y)$ if $\left|V\left(\sigma_{z}\right)\right| \leqslant$ $\left|V\left(\sigma_{y}\right)\right|$. (In particular, if $\left|V\left(\sigma_{y}\right)\right|=\left|V\left(\sigma_{z}\right)\right|$, then both arcs are added.)

We observe that $A_{1}$ satisfies (1)-(3). Since neither the vertices nor the labels were changed, the only thing that we need to show is that if the arc $(y, z)$ was added, then for all sequences $\left(T_{1}, \ldots, T_{t}\right) \in \cap_{(i, j) \in J} \Lambda_{(i, j)}$ and all $\left(\left(\sigma_{i}, w_{i}\right),\left(\sigma_{k}, w_{k}\right)\right) \in \ell(y) \times \ell(z)$, it holds that $T_{i} \subseteq T_{k}$. This follows from Observation 3.1. since $(y, z)$ was added, there exists $s \in\{1, \ldots, t\}$ such that $T_{s}$ is contained in both $T_{i}$ and $T_{k}$.

The directed graph $A_{2}$ is obtained from $A_{1}$ by recursively contracting all directed cycles of $A_{1}$. Specifically, for each directed cycle $C$, all the vertices of $C$ are contracted into a vertex $z_{C}$ (parallel arcs are removed, but not directed cycles of length 2$)$, and $\ell\left(z_{C}\right):=\cup_{x \in V(C)} \ell(x)$. We again observe that $A_{2}$ satisfies properties (1)-(3).

Finally, $A_{3}$ is obtained from $A_{2}$ by recursively deleting transitivity arcs, that is, the arc $(y, z)$ is removed if there exists a directed path of length greater than 1 from $y$ to $z$. Note that $A_{2}$ and $A_{3}$ have the same vertex-set, and every arc of $A_{3}$ is also an arc in $A_{2}$. Again, $A_{3}$ readily satisfies properties (1)-(3).

Now, let us prove that each component of $A_{3}$ is an arborescence, that is a directed acyclic graph with each out-degree at most one. We only need to show that every vertex of $A_{3}$ has outdegree at most 1 . Assume that $(x, y)$ and $(x, z)$ are two arcs of $A_{3}$. First, note that, in $A_{2}$, there is no directed path from $y$ to $z$ or from $z$ to $y$, for otherwise the arc $(x, y)$ or the arc $(x, z)$ would not belong to $A_{3}$, respectively. Therefore, regardless of whether $y$ and $z$ arose from contractions of directed cycles in $A_{1}$, there exist three vertices $x^{\prime}, y^{\prime}$ and $z^{\prime}$ in $A_{1}$ such that both $\left(x^{\prime}, y^{\prime}\right)$ and $\left(x^{\prime}, z^{\prime}\right)$ are arcs but neither $\left(y^{\prime}, z^{\prime}\right)$ nor $\left(z^{\prime}, y^{\prime}\right)$ is an arc. This contradicts the definition of $A_{1}$ Consequently, every vertex of $A_{3}$ has outdegree at most 1 , as wanted.

We define $\tau_{i}$ to be the sequence

$$
\left(\sharp\left(\left(\sigma_{i}, w_{i}\right) \hookrightarrow(T, w)\right), \sharp\left(\left(\sigma_{i}, w_{i}\right) \hookrightarrow\left(\sigma_{1}, w_{1}\right)\right), \ldots, \sharp\left(\left(\sigma_{i}, w_{i}\right) \hookrightarrow\left(\sigma_{t}, w_{t}\right)\right)\right)
$$

We recall that $\tau_{1}, \ldots, \tau_{t}$ are known from the assumptions of Procedure 1. Step 2 is completed by the following procedure.

\section{Procedure 2.}

INPUT: A labeled directed forest $A$ of arborescences and the sequences $\tau_{1}, \ldots, \tau_{t}$.

OUTPut: For each $H \in\left\{(T, w),\left(\sigma_{1}, w_{1}\right), \ldots,\left(\sigma_{t}, w_{t}\right)\right\}$, the number $\mathcal{P}_{3}(H, A, \tau(T))$ of elements $\left(T_{1}, \ldots, T_{t}\right)$ of $\Lambda_{0}(H)$ such that

- for each vertex $x$ of $A$, if $\left(\sigma_{i}, w_{i}\right),\left(\sigma_{k}, w_{k}\right) \in \ell(x)$ then $T_{i}=T_{k}$; and

- for every $\operatorname{arc}(x, y)$ of $A$, if $\left(\left(\sigma_{i}, w_{i}\right),\left(\sigma_{k}, w_{k}\right)\right) \in \ell(x) \times \ell(y)$, then $T_{i} \subseteq T_{k}$.

The output of Procedure 2 can be recursively computed as follows. Let $V_{\max }$ be the set of vertices of $A$ with outdegree 0 . For each vertex $x$ of $A$, let $\left(\sigma^{x}, w^{x}\right)$ be a representative of $\ell(x)$.

$$
\mathcal{P}_{3}(H, A, \tau(T))=\prod_{x \in V_{\max }}\left(\sharp\left(\left(\sigma^{x}, w^{x}\right) \hookrightarrow H\right)\right) \cdot \mathcal{P}_{3}\left(\left(\sigma^{x}, w^{x}\right), \tilde{A}(w), \tau(T)\right),
$$

where $\tilde{A}(w)$ is obtained from the component of $A$ that contains $x$ by removing $x$. 
By property (3) of the labels, the output $\mathcal{P}_{3}\left(T, A_{3}, \tau(T)\right)$ is equal to $\left|\cap_{(i, k) \in J} \Lambda_{(i, k)}\right|$. This concludes the design of Procedure 1 .

Acknowledgements. The authors thank the referees for their numerous suggestions on a preliminary version of this work, including pointing out some inaccuracies.

\section{REFERENCES}

[1] J. Aliste-Prieto and J. Zamora, Proper caterpillars are distinguished by their chromatic symmetric function. Discrete Math. 315 (2014), 158-164. https://doi.org/10.1016/j.disc.2013.10.016

[2] J. D. Annan, Complexity of Counting Problems. Ph.D. thesis. Oxford University, 1994.

[3] A. Brandstädt, V. B. Le, and J. P. Spinrad, Graph classes: a survey. SIAM Monographs on Discrete Mathematics and Applications. Society for Industrial and Applied Mathematics (SIAM), Philadelphia, PA, 1999. https://doi.org/10.1137/1.9780898719796

[4] T. Brylawski, Intersection theory for graphs. J. Combin. Theory Ser. B 30 (1981), no. 2, 233-246. https: //doi.org/10.1016/0095-8956(81) 90068-X

[5] J. Fougere, On symmetric chromatic polynomials of trees. Undergraduate thesis. Dartmouth College, 2003.

[6] S. Hell and C. Ji, On an Algorithm for Comparing the Chromatic Symmetric Functions of Trees. 2018. arXiv:1801.07363

[7] M. Klazar, M. Loebl, and I. Moffatt, The Potts model and chromatic functions of graphs. Ann. Inst. Henri Poincaré D 1 (2014), no. 1, 47-60. https://doi.org/10.4171/AIHPD/2

[8] M. Loebl, Chromatic polynomial, $q$-binomial counting and colored Jones function. Adv. Math. 211 (2007), no. 2, 546-565. https://doi.org/10.1016/j.aim.2006.09.001

[9] J. L. Martin, M. Morin, and J. D. Wagner, On distinguishing trees by their chromatic symmetric functions. J. Combin. Theory Ser. A 115 (2008), no. 2, 237-253. https://doi.org/10.1016/j.jcta.2007.05.008

[10] M. Morin, Caterpillars, ribbons, and the chromatic symmetric function. MS thesis. University of British Columbia, 2005.

[11] S. D. Noble and D. J. A. Welsh, A weighted graph polynomial from chromatic invariants of knots. Ann. Inst. Fourier (Grenoble) 49 (1999), no. 3, 1057-1087. Symposium à la Mémoire de François Jaeger (Grenoble, 1998). http://www.numdam.org/item?id=AIF_1999_-49_3_1057_0

[12] R. Orellana and G. Scott, Graphs with equal chromatic symmetric functions. Discrete Math. 320 (2014), 1-14. https://doi.org/10.1016/j.disc.2013.12.006

[13] J. Oxley and G. Whittle, Tutte invariants for 2-polymatroids. In Graph structure theory (Seattle, WA, 1991), Contemp. Math., 147. Amer. Math. Soc., Providence, RI, 1993, 9-19 https://doi.org/10.1090/conm/147/ 01163

[14] I. Sarmiento. Personal communication to S. D. Noble and D. J. A. Welsh. 1999.

[15] I. Smith, Z. Smith, and P. Tian, Symmetric Chromatic Polynomial of Trees. 2015. arXiv:1505.01889

[16] R. P. Stanley, A symmetric function generalization of the chromatic polynomial of a graph. Adv. Math. 111 (1995), no. 1, 166-194.https://doi.org/10.1006/aima.1995.1020

[17] R. P. Stanley, Graph colorings and related symmetric functions: ideas and applications: a description of results, interesting applications, \& notable open problems. Discrete Math. 193 (1998), no. 1-3, 267-286. Selected papers in honor of Adriano Garsia (Taormina, 1994). https://doi.org/10.1016/S0012-365X (98)00146-0

[18] J. Zamora, Structural Graph Theory. Ph.D. thesis. Charles University, Prague, 2013.

Dept. of Applied Mathematics, Charles University, Praha, Czech Republic.

E-mail address: loebl@kam.mff.cuni.cz

Centre National de la Recherche Scientifique, (CStB), Strasbourg, France.

E-mail address: sereni@kam.mff.cuni.cz 